\title{
Compostos fenólicos e capacidade antioxidante em frutos de tomateiros mutantes fotomorfogenéticos
}

\section{Phenolic compounds and antioxidant capacity in fruits of photomorphogenic mutant tomato}

\author{
Tiago Dias ${ }^{I}$ Hyrandir Cabral de Melo $^{I *}$ Frederico Rocha Rodrigues Alves $^{\text {II }}$ \\ Rogério Falleiros Carvalho ${ }^{\mathrm{III}}$ Karla da Silva Carneiro ${ }^{\mathrm{IV}}$ Cleiton Mateus Sousa $^{\mathrm{IV}}$
}

\section{RESUMO}

Compostos fenólicos e capacidade antioxidante são mecanismos de defesa das plantas aos danos do estresse oxidativo. Os compostos fenólicos são sintetizados pela via dos fenilpropanoides, cuja enzima chave, fenilalanina amônia liase, é influenciada pela luz e ação de fotorreceptores, como o fitocromo. $O$ objetivo do presente trabalho é avaliar a concentração de compostos fenólicos e a capacidade antioxidante de frutos de microtomateiro selvagem, cultivar "Micro-Tom" (MT), e seus mutantes fotomorfogenéticos high pigment 1 (hp1), superresponsivo a eventos mediados por luz e aurea (au), deficiente quantitativo em fitocromos. Vinte frutos maduros de cada genótipo (MT, hp1 e au) foram utilizados para as análises, realizadas em triplicata. Para quantificação dos compostos fenólicos totais, foi utilizado o método de Folin-Ciocalteu e a capacidade antioxidante foi realizada pelos métodos Ferric Reducing Antioxidant Power (FRAP) e 2,2-diphenyl-1-picrylhydrazyl (DPPH). Os frutos do mutante hp1 apresentaram maiores conteúdos de compostos fenólicos totais e também maior capacidade antioxidante em relação à cultivar selvagem ("MT") e ao mutante au, o qual não diferiu significativamente da cultivar " $M T$ ".

Palavras-chave: aurea, fitocromo, hp1, Solanum lycopersicum L.

\section{ABSTRACT}

Phenolic compounds and antioxidant capacity are defense mechanisms of plants against the oxidative stress damage. Phenolic compounds are synthesized through the phenylpropanoid pathway, where the enzyme phenylalanine-ammonia-lyase plays a key role and it is influenced by light and photoreceptors such as phytochromes. The present research aims to evaluate the phenolic compounds content and antioxidant capacity of the wild "MicroTom" (MT) cultivar tomato fruits and its photomorphogenic mutant tomato plants high pigment 1 (hp1), super responsive to events mediated by light, and aurea (au), quantitative phytochrome deficient. Twenty mature fruits of each genotype ("MT", hp1, au) were used in triplicate for analyses. To quantify the total phenolic compounds the Folin-Ciocalteu method was used and the antioxidant capacity was analyzed by Ferric Reducing Antioxidant Power (FRAP) and 2,2-diphenyl-1-picrylhydrazyl (DPPH) methods. The hpl mutant presented the highest total phenolic compounds content and higher antioxidant capacity than wild cultivar ("MT") and au mutant, which did not differ significantly from " $M T$ " cultivar.

Key words: aurea, hp, phytochrome, Solanum lycopersicum L.

\section{INTRODUÇÃO}

Substâncias antioxidantes são aquelas que, quando presentes em baixas concentrações, comparativamente ao conjunto daquelas presentes em um substrato oxidável, atrasam ou previnem de forma significativa a oxidação deste substrato. Do ponto de vista nutricional, antioxidantes alimentares, particularmente em vegetais, têm ganhado um grande interesse entre os consumidores e a comunidade científica, uma vez que estudos têm associado menor risco de desenvolvimento de doenças cardiovasculares e câncer ao consumo destes compostos (TEMPLE, 2000). Em tecidos vegetais, estas biomoléculas podem ser enzimas, vitaminas, carotenoides e compostos fenólicos (HALLIWELL \& GUTTERIDGE, 1995).

'Laboratório de Nutrição e Análise de Alimentos, Universidade Federal de Goiás (UFG), Goiânia, GO, Brasil.

ILLaboratório de Fisiologia Vegetal, UFG, Campus Samambaia, 74001-970, Goiânia, GO, Brasil, E-mail: hyrandir@yahoo.com.br.

*Autor para correspondência.

IIIFaculdade de Ciências Agrárias e Veterinária, Universidade Estadual Paulista (UNESP), Campus Jaboticabal, SP, Brasil.

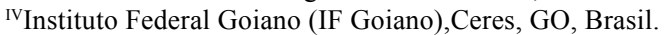


Os compostos fenólicos constituem um grupo diverso de metabólitos secundários que apresentam atividade antioxidante, como flavonoides e taninos. Estes compostos constituem a classe mais abundante de metabólitos secundários nas plantas e compartilham uma origem em comum na via biossintética dos fenilpropanoides (GRACE \& LOGAN, 2000).

O metabolismo dos fenilpropanoides tem a fenilalanina amônia-liase (PAL) como uma enzima chave, responsável por catalisar a conversão da fenilalanina em ácido transcinâmico, o primeiro passo para a biossíntese dos fenilpropanoides. A atividade da PAL parece ser extraordinariamente sensível ao estado fisiológico da planta. Dentre os fatores ambientais, as mudanças na atividade da PAL podem ocorrer por ação da luz. A atividade da PAL é estimulada pelo comprimento de onda do vermelho, e reprimida pelo comprimento de onda do vermelho distante (CAMM \& TOWERS, 1973), resposta tipicamente mediada pelo fotorreceptor fitocromo e observada em tomate, arroz e milho (ZUCKER, 1972; GUO \& WANG, 2009).

Fitocromos são fotorreceptores relacionados com a percepção qualitativa e quantitativa da luz pelas plantas, desencadeando diversas e complexas respostas fisiológicas. Descobertas recentes têm demonstrado que estas moléculas também estão relacionadas com uma gama de respostas ao estresse abiótico e biótico, devido ao seu papel na regulação da transcrição de genes específicos, influenciando mecanismos bioquímicos e moleculares de sinalização celular (CARVALHO et al., 2011). A elucidação dos processos fisiológicos dependentes dos fitocromos tem sido possível por meio de mutantes específicos, tanto para a biossíntese de fotorreceptores quanto para a via de transdução de sinal desencadeada por eles. Em tomateiros (Solanum lycopersicum L.), o mutante aurea (au) apresenta deficiência quantitativa em todos os fitocromos (TERRY \& KENDRICK, 1996), enquanto o mutante high pigment1 ( $h p l)$ é super-responsivo a eventos mediados por luz (KENDRICK et al., 1997).

O presente trabalho tem como objetivo avaliar o conteúdo de compostos fenólicos e a capacidade antioxidante em frutos de microtomateiros mutantes fotomorfogenéticos au e $h p 1$, bem como sua cultivar selvagem 'Micro-Tom' (MT).

\section{MATERIAL E MÉTODOS}

Microtomateiros da cultivar selvagem 'Micro-Tom' (MT), usada como controle neste experimento, e mutantes fotomorfogenéticos au e $h p 1$ foram cultivados em casa de vegetação entre setembro de 2012 a janeiro de 2013 no Instituto Federal Goiano, Ceres - GO, localizado a $15^{\circ} 18^{\prime} 30^{\prime \prime}$ $\mathrm{S}$ e $49^{\circ} 35^{\prime} 54^{\prime}$ "W. As plantas foram cultivadas em potes plásticos com capacidade de $1 \mathrm{~L}$ de substrato, contendo terra escura comercial, suplementadas com $5 \mathrm{~g}$ de NPK 4-14-8, regadas diariamente e submetidas a um fotoperíodo de 12 horas.

Ao atingirem o estágio de amadurecimento, os frutos de 20 plantas de cada genótipo (MT, au, $h p 1)$ foram colhidos e armazenados em freezer a $-18^{\circ} \mathrm{C}$. As análises foram realizadas posteriormente no Laboratório de Nutrição e Análise de Alimentos da Faculdade de Nutrição da Universidade Federal de Goiás.

Os frutos foram submetidos a um processador de alimentos doméstico e separados em amostras para liofilização e posterior análise de compostos fenólicos totais e capacidade antioxidante. As amostras foram submetidas a um liofilizador (Liotop, modelo L 101) por 72 horas a $-55^{\circ} \mathrm{C}$ e pressão de $1000 \mu \mathrm{mHg}$. O material liofilizado obtido foi triturado em micro-moinho analítico (IKA, modelo A11 basic) e o pó, armazenado em frascos de vidro com tampa em freezer a $-18^{\circ} \mathrm{C}$ até o momento das análises.

Para a determinação de compostos fenólicos totais e da capacidade antioxidante, as amostras liofilizadas em pó foram extraídas com metanol $70 \%$. Para isso, foram pesados $0,5 \mathrm{~g}$ de cada amostra, em triplicata, e foi adicionado $20 \mathrm{~mL}$ de metanol 70\%. Agitou-se em placa de agitação magnética por $2 \mathrm{~h}$, filtrou-se em papel de filtro e transferiu-se o filtrado para balão volumétrico de $50 \mathrm{~mL}$, completando o volume com metanol $70 \%$.

Para determinação de compostos fenólicos totais, foi utilizada a metodologia de SINGLETON \& ROSSI (1965). Determinou-se a capacidade antioxidante pelo método Ferric Reducing Antioxidant Power (FRAP) (BENZIE \& STRAIN, 1996) e pelo método 2,2-diphenyl-1-picrylhydrazyl (DPPH) (BRAND-WILLIAMS et al., 1995).

O experimento foi conduzido em delineamento inteiramente casualizado. Todas as análises foram realizadas em triplicata e os dados foram submetidos à análise de variância e separados em classes distintas, utilizando-se o teste de Tukey em nível de erro de 5\%.

\section{RESULTADOS E DISCUSSÃO}

Os frutos do mutante $h p l$ apresentaram maior conteúdo de compostos fenólicos, 
comparativamente à cultivar selvagem ' $\mathrm{MT}$ ' e ao mutante $a u$ (Figura 1). O mutante $h p l$ também deteve maior capacidade antioxidante, comparativamente à cultivar 'MT' e ao mutante $a u$, tanto avaliada pelo método de redução de íon férrico, FRAP (BENZIE \& STRAIN, 1996), quanto avaliada por método de sequestro de radicais livres, DPPH (BRAND-WILLIAMS et al., 1995) (Figura 1). Não foram observadas diferenças significativas entre a cultivar 'MT' e o mutante au tanto para conteúdo de compostos fenólicos quanto para capacidade antioxidante (Figura 1).

O maior conteúdo de compostos fenólicos observado no mutante $h p 1$, provavelmente tenha ocorrido em função da regulação positiva da luz sobre proteínas envolvidas na rota biossintética desses compostos. A mutação de $h p l$ interfere na via de transdução de sinais, conferindo uma hipersensibilidade das plantas aos eventos mediados por luz, tornandoas super-responsivas a este fator (KENDRICK et al., 1997), a qual é uma resposta mediada pelos fitocromos. Por sua vez, a via biossintética dos compostos fenólicos é regulada predominantemente pela enzima
PAL (WEISSHAAR \& JENKINS, 1998; CHANG et al., 2008; GHOLIZADEH \& KOHNEHROUZ, 2010), a qual é uma enzima reconhecidamente regulada pelo padrão de radiação incidente na planta e, consequentemente, pelos fitocromos (SCHOPFER, 1977; GILIBERTO et al., 2005). Embora trabalhos anteriores já tenham comprovado que a regulação de proteínas da via biossintética dos compostos fenólicos seja regulada por padrões específicos de radiação, os resultados deste trabalho comprovam que a mutação na via de transdução de sinais em $h p 1$, embora este não seja considerado um mutante quantitativo em fitocromos (KENDRICK et al., 1997), é responsável quanto à regulação da via biossintética dos compostos fenólicos.

É esperado que tomates do mutante $h p 1$, devido a suas respostas consideradas exageradas à luz, tenham características fotodependentes parecidas aos frutos expostos ao sol durante seu crescimento ou aos frutos de plantas cultivadas a pleno sol, comparativamente às plantas cultivadas à sombra. Em uvas, houve aumento tanto para a atividade da PAL quanto para o conteúdo de compostos fenólicos,

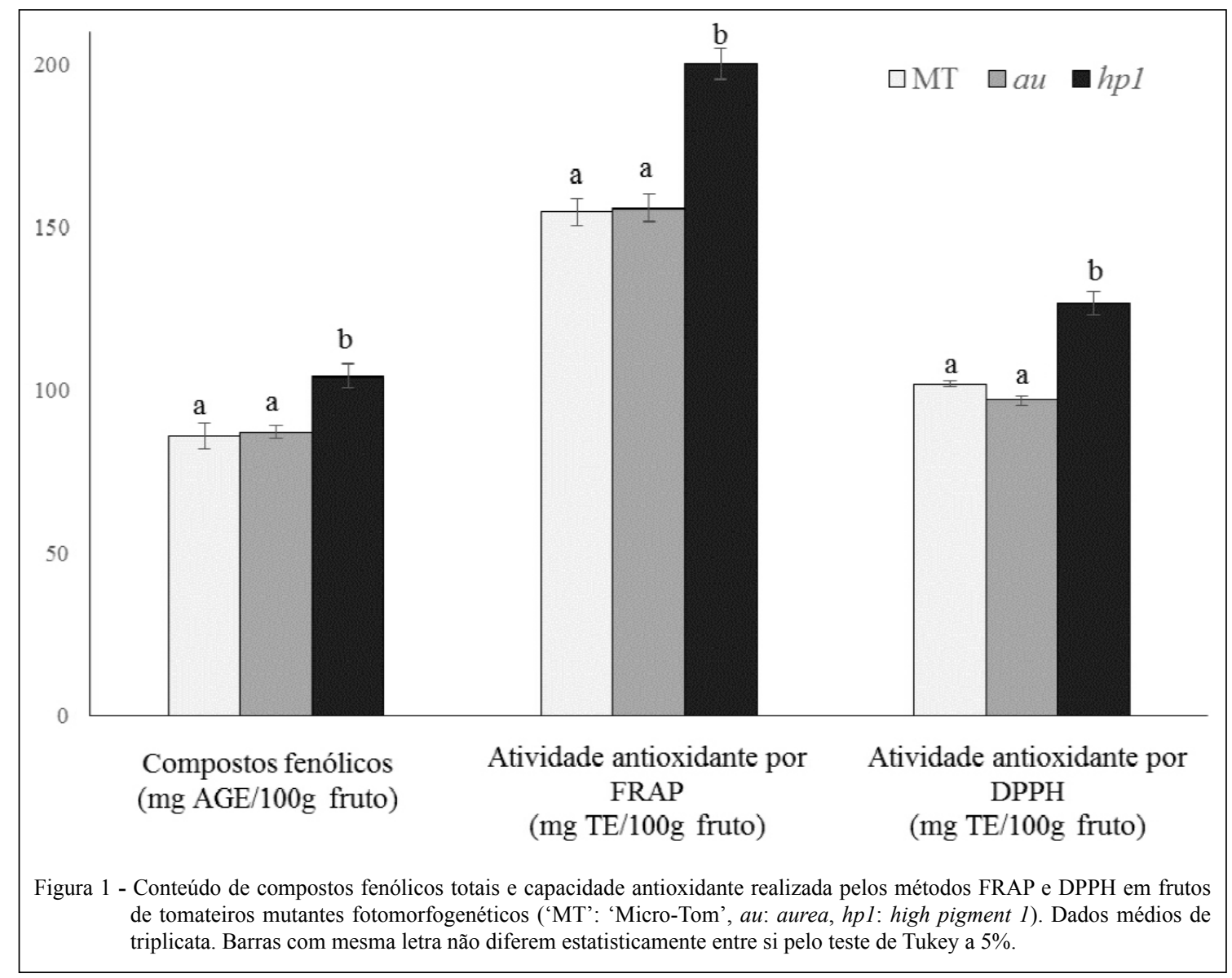

Ciência Rural, v.45, n.5, mai, 2015. 
quando as plantas foram cultivadas a pleno sol (KLIEWER, 1977; ROUBELAKIS-ANGELAKIS \& KLIEWER, 1986). Esse aumento também ocorreu quando foi analisado o conteúdo de compostos fenólicos em frutos de kiwi e maçã, expostos ao sol durante o seu crescimento, em relação àqueles expostos à sombra (MONTANARO et al., 2007; FENG et al., 2013). Estas observações corroboram as expectativas geradas e confirmadas neste trabalho para o conteúdo de compostos fenólicos em frutos do tomateiro mutante $h p l$.

O maior conteúdo de compostos fenólicos ou na capacidade antioxidante, tal qual observado para frutos do mutante $h p l$ neste trabalho, ocorre como forma de defesa de tecidos vegetais a condições que favoreçam o aumento de radicais livres oriundos do metabolismo celular, em organelas, como cloroplastos, mitocôndrias e peroxissomos (DEL RIO et al., 2006; NAVROT et al., 2007). Os radicais livres se acumulam especialmente em condições ambientais indutoras de estresse, como o excesso de luminosidade (APEL \& HIRT, 2004; GILL \& TUTEJA, 2010; WITUSZVNSKA \& KARPINSKI, 2013), seca, salinidade, patógenos e poluentes atmosféricos (MITTLER， 2002; GILL \& TUTEJA， 2010, SHARMA et al., 2012). Essa resposta diferenciada do mutante $h p 1$, em detrimento dos demais genótipos, provavelmente tenha ocorrido devido a sua maior suscetibilidade a estresse, promovido por excesso de luminosidade, devido a sua característica comum de hipersensibilidade às respostas mediadas por luz (KENDRICK et al., 1997), seja pela necessidade de maior reparação de danos provocado pelo estresse ou seja de forma preventiva.

Em frutos do mutante aurea, observou-se um conteúdo de compostos fenólicos e capacidade antioxidante similar à cultivar 'MT' (Figura 1), apesar da mutação em aurea ser caracterizada por uma menor quantidade de fitocromos e pigmentos fotossintéticos na planta (MURAMOTO et al., 2005; TERRY \& KENDRICK, 1996), condição que poderia levar à uma menor sensibilidade das plantas a reações desencadeadas por luz, o que não se traduziu em menor conteúdo de compostos fenólicos e capacidade antioxidante em seus frutos (Figura 1). O fato dos frutos do mutante aurea não possuir menor conteúdo de compostos fenólicos ou capacidade antioxidante em detrimento da deficiência de aparato fotorreceptor de suas plantas, quando comparado à cultivar selvagem 'MT', deve-se à possibilidade de essa mutação ser mais efetiva em provocar alterações em órgãos vegetativos ou ainda pode ter sido influenciado pelo fato de a planta mutante apresentar os efeitos de sua mutação de forma mais proeminente em seu estágio inicial de desenvolvimento, comparativamente à planta adulta (BECKER et al., 1992), estágio no qual ocorre a produção de frutos.

Maior conteúdo de compostos fenólicos, carotenoides e algumas vitaminas de potencial antioxidante e de interesse ao consumo humano, observados em folhas do mutante hpl (JARRET et al., 1984), necessariamente não está associado a um aumento na atividade de enzimas antioxidantes de interesse fotoprotetor da planta como catalase e peroxidase, que tiveram menor atividade em folhas de $h p 1$, quando comparado à cultivar selvagem, mesmo com aumento significativo no conteúdo de peróxido de hidrogênio observado neste mutante. Diferentemente do mutante aurea, que deteve maiores atividades destas enzimas antioxidativas, quando comparado ao mutante $h p l$, mesmo com conteúdo de peróxido de hidrogênio inferior ao mutante $h p l$ (MONTEIRO et al., 2012). Nesse sentido, podese inferir que, embora não tenham sido observadas, neste trabalho, alterações significativas no conteúdo de compostos fenólicos e capacidade antioxidante em frutos do mutante aurea, quando comparado aos da cultivar selvagem 'MT' (Figura 1), não se descarta a influência desta mutação sobre outros componentes do aparato antioxidativo não contemplados pelas análises efetuadas neste trabalho.

\section{CONCLUSÃO}

O conteúdo de compostos fenólicos e a capacidade antioxidante aumentam em tomates do mutante $h p 1$, mas não são alterados em tomates do mutante aurea, quando comparados à cultivar selvagem 'MT'.

\section{REFERÊNCIAS}

APEL, K.; HIRT, H. Reactive oxygen species: metabolism, oxidative stress and signal transduction. Annual Review of Plant Biology, v.55 p.373-399, 2004. Disponível em: $<$ http://www.annualreviews.org/doi/pdf/10.1146/annurev. arplant.55.031903.141701>. Acesso em: 13 jan. 2014. doi: 10.1146/annurev.arplant.55.031903.141701.

BECKER, T.W. et al. Light-regulated expression of the nitratereductase and nitrite-reductase genes in tomato and in the phytochrome-deficient aurea mutant of tomato. Planta, v.188, p.39-47, 1992. Disponível em: <http://link.springer.com/ article/10.1007\%2FBF00198937>. Acesso em: 21 jan. 2014.

BENZIE, I.F.F.; STRAIN, J.J. The ferric reducing ability of plasma (FRAP) as a measure of antioxidant power: the FRAP essay. Analytical Biochemistry, v.239, n.1, p.70-76, 1996. Disponível em: <http://www.sciencedirect.com/science/article/pii/ S0003269796902924>. Acesso em: 21 jan. 2014. 
BRAND-WILLIAMS, W. et al. Use of a free radical method to evaluate antioxidant activity. Food Science and Technology, v.28, n.1, p.25-30, 1995. Disponível em: <http://www.sciencedirect. com/science/article/pii/S0023643895800085>. Acesso em: 19 jan. 2014

CAMM, E.L.; TOWERS, G.H.N. Phenyalanine ammonia lyase. Phytochemistry, v.12, n.5, p.961-973, 1973. Disponível em: $<$ http:// www.sciencedirect.com/science/article/pii/0031942273850010>. Acesso em: 21 jan. 2014.

CARVALHO, R.F. et al. The role of phytochrome in stress tolerance. Journal of Integrative Plant Biology, v.53, n.12, p.920-929, 2011. Disponível em: $<$ http://onlinelibrary.wiley.com/ doi/10.1111/j.1744-7909.2011.01081.x/pdf >. Acesso em: 21 jan. 2014. doi: 10.1111/j.1744-7909.2011.01081.x.

CHANG, A. et al. Tomato phenylalanine ammonia-lyase gene family, highly redundant but strongly underutilized. Journal of Biological Chemistry, v.283, n.48, p.33591-33601, 2008. Disponível em: <http://www.ncbi.nlm.nih.gov/pmc/articles/ PMC2662276/>. Acesso em: 19 jan. 2014. doi: 10.1074/jbc. M804428200.

DEL RIO, L.A. et al. Reactive oxygen species and reactive nitrogen species in peroxisomes: production, scavenging and role in cell signalling. Plant Physiology, v.141, p.330-335, 2006. Disponível em: <http://www.plantphysiol.org/content/141/2/330>. Acesso em: 21 jan. 2014. doi: 10.1104/pp.106.078204.

FENG, F. et al. Phenylpropanoid metabolites and expression of key genes involved in anthocyanin biosynthesis in the shaded peel of apple fruit in response to sun exposure. Plant Physiology and Biochemistry, v.69, p.54-61, 2013. Disponível em: <http:// www.sciencedirect.com/science/article/pii/S0981942813001587>. Acesso em: 21 jan. 2014. doi: 10.1016/j.plaphy.2013.04.020.

GHOLIZADEH, A.; KOHNEHROUZ, B.B. Activation of phenylalanine ammonia lyase as a key component of the antioxidative system of salt-challenged maize leaves. Brazilian Journal of Plant Physiology, v.22, n.4, p.217-223, 2010. Disponível em: <http://www.scielo.br/scielo.php?pid=S167704202010000400001\&script=sci_abstract $>$. Acesso em: 21 jan. 2014. doi: 10.1590/S1677-04202010000400001.

GILIBERTO, L. et al. Manipulation of the blue light photoreceptor cryptochrome 2 in tomato affects vegetative development, flowering time and fruit antioxidant content. Plant Physiology, v.137, p.199-208, 2005. Disponível em: <http://www.plantphysiol. org/content/137/1/199>. Acesso em: 21 jan. 2014. doi: 10.1104/ pp.104.051987.

GILL, S.S.; TUTEJA, N. Reactive oxygen species and antioxidant machinery in abiotic stress tolerance in crop plants. Plant Physiology and Biochemistry, v.48, p.909-930, 2010. Disponível em: <http://www.sciencedirect.com/science/article/pii/ S0981942810001798>. Acesso em: 21 jan. 2014. doi: 10.1016/j. plaphy.2010.08.016.

GRACE, S.C.; LOGAN, B.A. Energy dissipation and radical scavenging by the plant phenylpropanoid pathway. Philosophical Transactions of the Royal Society of London, v.355, p.14991510, 2000. Disponível em: <http://www.ncbi.nlm.nih.gov/pmc/ articles/PMC1692864/pdf/11128003.pdf>. Acesso em: 21 jan. 2014. doi: $10,1098 /$ rstb.2000.0710.
GUO, J.; WANG, M.H. Characterization of the phenylalanine ammonia-lyase gene (SIPAL5) from tomato (Solanum lycopersicum L.). Molecular Biology Reports, v.36, p.15791585, 2009. Disponível em: <http://link.springer.com/article/10 .1007\%2Fs11033-008-9354-9>. Acesso em: 21 jan. 2014. doi: $10.1007 /$ s11033-008-9354-9.

HALLIWELL, B.; GUTTERIDGE, J.M.C. Free radicals biology and medicine. Oxford: Oxford University, 1995. 346p.

JARRET, R.L. et al. Pleiotropic effects associated with the chlorophyll intensifier mutations high pigment and dark green in tomato. American Society for Horticultural Science, v.109, p.873-878, 1984.

KENDRICK, R.E. et al. Photomorphogenic mutants of tomato. Plant Cell and Environment, v.20, p.746-751, 1997. Disponível em: <http://onlinelibrary.wiley.com/doi/10.1046/j.1365-3040.1997. d01-109.x/abstract>. Acesso em: 21 jan. 2014. doi: 10.1046/j.13653040.1997.d01-109.x.

KLIEWER, W.M. Influence of temperature, solar radiation and nitrogen on coloration and composition of Emperor grapes. American Journal of Enology and Viticulture, v.28, p.96-103, 1977. Disponível em: <http://ajevonline.org/content/28/2/96>. Acesso em: 21 jan. 2014.

MITTLER, R. Oxidative stress, antioxidants and stress tolerance. Trends in Plant Science, v.7, n.9, p.405-410, 2002. Disponível em: < http://www.sciencedirect.com/science/article/pii/ S1360138502023129>. Acesso em: 21 jan. 2014. DOI: 10.1016/ S1360-1385(02)02312-9

MONTANARO, G, et al. Phenolic compounds in young developing kiwifruit in relation to light exposure: implications for fruit calcium accumulation. Journal of Plant Interactions, v. 2, n.1, p. 63-69, 2007. Disponível em: <http://www.tandfonline.com/ doi/pdf/10.1080/17429140701429228>. Acesso em: 21 jan. 2014. doi: $10.1080 / 17429140701429228$.

MONTEIRO, C.C. et al. Biochemical and histological characterization of tomato mutants. Anais da Academia Brasileira de Ciências, v.84, n.2, p.573-585, 2012. Disponível em: < http://www.scielo.br/pdf/aabc/v84n2/aop2212.pdf $>$. Acesso em: 21 jan. 2014. doi:10.1590/S0001-37652012005000022.

MURAMOTO, T. et al. The tomato photomorphogenic mutant aurea is deficient in phytochromobilin synthase for phytochrome chromophore biosynthesis. Plant \& Cell Physiology, v.46, n.4, p.661-665, 2005. Disponível em: <http://pcp.oxfordjournals.org/ content/46/4/661>. Acesso em: 21 jan. 2014. doi: 10.1093/pcp/ pci062.

NAVROT, N. et al. Reactive oxygen species generation and antioxidant systems in plant mitochondria. Physiologia Plantarum, v.129, p.185-195, 2007. Disponivel em: <http://onlinelibrary. wiley.com/doi/10.1111/j.1399-3054.2006.00777.x/pdf>. Acesso em: 21 jan. 2014. doi: 10.1111/j.1399-3054.2006.00777.x.

ROUBELAKIS-ANGELAKIS, K.A.; KLIEWER, W.M. Effects of exogenous factors on phenylalanine ammonia-lyase activity and accumulation of anthocyanins and total phenolics in grape berries. American Journal of Enology and Viticulture, v.37, p.275-280, 1986. Disponível em: <http://www.ajevonline.org/ content/37/4/275>. Acesso em: 21 jan. 2014. 
SCHOPFER, P. Phytochrome control of enzymes. Annual Review of Plant Physiology, v.28, p.223-252, 1977. Disponível em: <http://www.annualreviews.org/doi/pdf/10.1146/annurev. pp.28.060177.001255>. Acesso em: 21 jan. 2014.

SHARMA, P. et al. Reactive oxygen species, oxidative damage and antioxidative defense mechanism in plants under stressful conditions. Journal of Botany, v.2012. Article ID 217037. 26p., 2012. Disponível em: <http://www.hindawi. com/journals/jb/2012/217037/>. Acesso em: 22 jan. 2014.doi: $10.1155 / 2012 / 217037$.

SINGLETON, V.L.; ROSSI, J.A. Colorimetry of total phenolics with phosphomolybidic-phosphotungstic acid reagent. American Journal of Enology and Viticulture, v.16, p.144-158, 1965 Disponível em: <http://ajevonline.org/content/16/3/144>. Acesso em: 22 jan. 2014

TEMPLE, N.J. Antioxidants and disease: More questions than answers. Nutrition Research, v.20, n.3, p.449-459, 2000. Disponível em: <http://www.sciencedirect.com/science/article/pii/ S027153170000138X > . Acesso em: 22 jan. 2014. doi: 10.1016/ S0271-5317(00)00138-X.
TERRY, M.J.; KENDRICK, R.E. The aurea and yellowgreen-2 mutants of tomato are deficient in phytochrome chromophore synthesis. Journal of Biological Chemistry, v.271, n.35, p.21681-21686, 1996. Disponível em: <http://www.jbc.org/ content/271/35/21681.full>. Acesso em: 22 jan. 2014 . doi: 10.1074/jbc.271.35.21681.

WEISSHAAR, B.; JENKINS, GI. Phenylpropanoid biosynthesis and its regulation. Current Opinion in Plant Biology, v.1, n.3, p.251-257, 1998. Disponível em: <http://www.sciencedirect. com/science/article/pii/S1369526698801131>. Acesso em: 22 jan. 2014. doi: 10.1016/S1369-5266(98)80113-1.

WITUSZVNSKA, W.; KARPINSKI S. Programmed cell death as a response to high light, UV and drought stress in plants. In: VAHDATI, K.; LESLIE, C. Abiotic stress: plant responses and applications in agriculture. Croacia: InTech, 2013. 410p.

ZUCKER, M. Light and enzymes. Annual Review of Plant Physiology, v.23, p.133-156, 1972. Disponível em: $<$ http://www.annualreviews.org/doi/pdf/10.1146/annurev. pp.23.060172.001025>. Acesso em: 22 jan. 2014. 